\title{
Rotordynamic Characteristics Analysis for an aero-engine Low Pressure Rotor Rig Test Model
}

\author{
Huiying Song ${ }^{1, a}$, Shaohui Wang ${ }^{1}$ and Kai Sun ${ }^{1}$ \\ ${ }^{1}$ AECC Commercial Aircraft Engine Co., Ltd., South Lianhua Road 3998, 201108, Shanghai, China
}

\begin{abstract}
D whole engine finite element model of a low pressure rotor rig test model is established in this paper. Rotorynamic characteristics are mainly analysed with three models, which include rotor model with static stiffness, rotor model with dynamic stiffness, and whole engine model. The calculation results were compared and discussed deeply. Rotordynamic characteristics of rotor model with static stiffness are similar with the rotor model with dynamic stiffness, but the latter may have additional resonance peaks caused by dynamic stiffness. Whole engine model, which can capture the modes of casing and coupling vibration between stator and rotor, may have more critical speeds than rotor model only. The unbalance response amplitude and phase angle of the whole engine model are different with the only rotor model, in the values and distributions of the peaks. The result of rotor model with dynamic stiffness is closed to the whole engine model than the rotor model with static stiffness. The peak values of the whole engine model are smaller than the only rotor model. Rotordynamic characteristics with whole engine model are more accurate than rotor model only, so it necessary to analyse rotordynamic characteristics with whole engine model in detail design stage.
\end{abstract}

\section{Introduction}

Finite element Aero-engine rotordynamic model usually does not include detailed casing model. Instead, it has been a common practice to represent casing using spring model. There are two types of spring casing models in traditional rotor dynamics analysis. One only considers static stiffness $K_{s}$ and the other considers dynamic stiffness $K_{d}$. In the $K_{s}$ method, no casing modes nor coupling modes between rotor and casing are considered. As for the $K_{d}$ method, casing modes are considered, and the spring constant calculation is similar to that for the static stiffness. The only difference is the force is applied with a frequency, in other words, the rotor support stiffness changes with rotor speed, especially near the stator resonant frequencies. Consequently, the frequencies in Campbell diagram change severely with rotor speed.

Thin shell structure casing is used in most modern aero-engines. Therefore, there are coupling effects between the dynamic characteristics of the rotor and the casing, which has to be properly characterized in rotordynamic analysis. The coupling effect cannot be properly reflected by the two methods mentioned above, but can be addressed by a 3D whole engine finite element model[1-5],

\footnotetext{
${ }^{\mathrm{a}}$ Corresponding author : songhy02@163.com
} 
including major engine structure characteristics and capturing the casing modes and coupled vibrations between the casing and the rotor.

In this paper, 3D finite element model of an aero-engine low pressure rotor rig test system is established. Rotordynamic characteristics are simulated using three models mentioned above. The results are compared and analysed, recommendations for the future studies are made in the conclusion.

\section{3D whole engine finite element modelling and Modal analysis}

The 3D whole engine finite element model of a low pressure rotor rig test model is shown in figure 1, which includes the casing and the rotor. The rotor is supported by 3 bearings. Both the rotor and casing are modelled using SOLID185 elements. The keyoption for the SOLID185, KEYOPT(2)=2 is chosen to overcome shear locking[6]. MASS21 elements are used to modelling blades, which is connected with disk by RBE3. The COMBI214 elements are used to modelling bearings and supports, which connect the rotor to the casing.

MEMF(Modal Effective Mass Fraction )[7], obtained by dividing the modal effective mass by the model total mass, is a common indicator for measuring the significance level of a mode. Therefore, a mode with a large effective mass, which is usually global mode, will contributes significantly to the system's response. Modal analyses are performed for both the casing and the engine. The global vibration mode shapes of casing and engine in frequency domain are demonstrated in Figures 3 and 4 respectively, where frequency ratio replaces the actual frequency. The global vibration mode shapes of the whole engine are shown in figure 4, including coupling modes between the rotor and the casing.
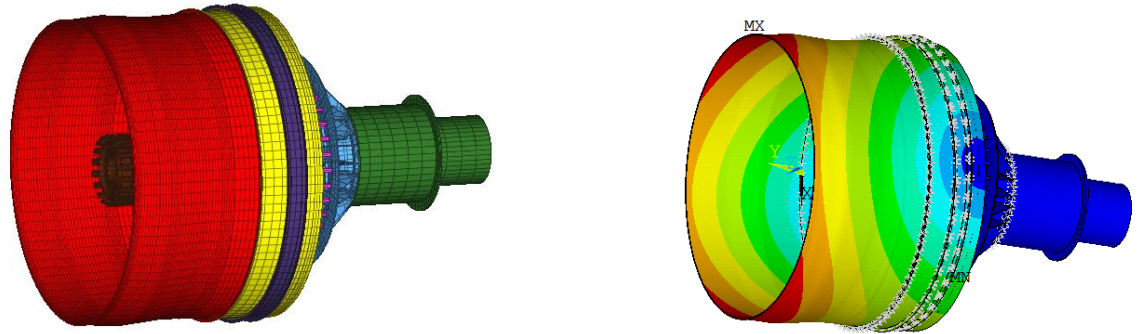

Figure 1. The 3D whole engine finite element model. Figure 2. Mode of casing (frequency ratio 0.40).

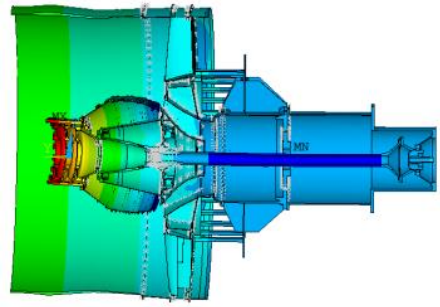

(a) mode 1 (frequency ratio 0.372 )

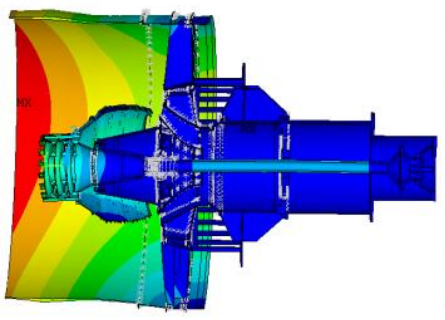

(b) mode 2 (frequency ratio 0.424 )

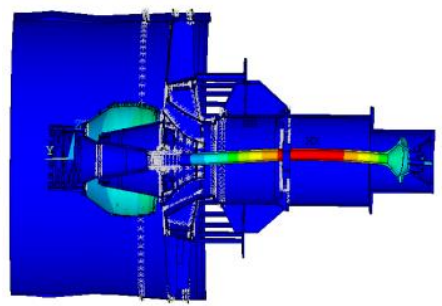

(c) mode 3 (frequency ratio 0.731 )

Figure 3. Modes of engine

\section{Static stiffness and dynamic stiffness}

If we look at the rotor model only, instead of the whole engine model, the static stiffness and dynamic stiffness can be determined by experiments or calculations[8,9]. The static and dynamic stiffness of the casing can be obtained by the linear static analysis and the harmonic response analysis respectively. Since the casing is axisymmetric, the stiffness in the two orthogonal directions is 
assumed to be same. The static stiffness and dynamic stiffness of the casing support as functions of frequency ratio are shown in figure 4 , with peak around 0.4 . The casing mode around frequency ratio at 0.4 is excited and the mode shape is shown in Figure 2. The mode is excited by the bearing excitations. Dynamic stiffness as a function of frequency, severely impacting the casing resonant frequencies.

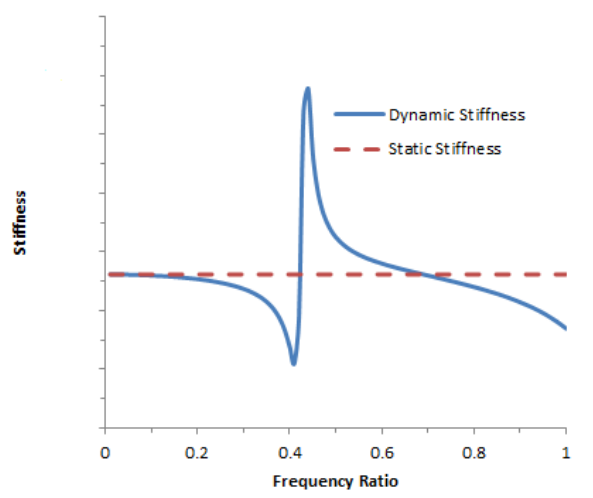

Figure 4. The static stiffness and dynamic stiffness of a support in the casing.

\section{Rotorynamic Characteristics}

\subsection{Rotorynamic Characteristics based on rotor model only with static stiffness}

In the $K_{s}$ method, only the stiffness but no modes of the casing is considered, which means only rotor modes are represented and no casing modes nor coupling between rotor and casing is considered. The Campbell diagram based on the static stiffness is shown in figure 5. Here the speed ratio is used to replace the actual speed. FW represents forward whirling, BW represents backward whirling. As demonstrated in Figure 6, there are two critical speeds in the rotor speed range, the first one (CS1) is 0.527 and the second one (CS2) is 0.760 . Figure 6 shows the mode shapes of the rotor (mode 1 and mode 2). The unbalance load is applied at the fan disk, and a harmonic force response analysis is performed in the frequency range of interest. The displacement response amplitude and phase angle are presented in the following figures 7 and 8 respectively. There are two distinct peaks in the exciting frequency range, the first distinct peak(peak1) and a sharp phase shift about 180 degree occur around 0.52, corresponding to CS1, the second distinct peak(peak2) with a sharp phase shift about 180 degree occur around 0.77 , corresponding to CS2.

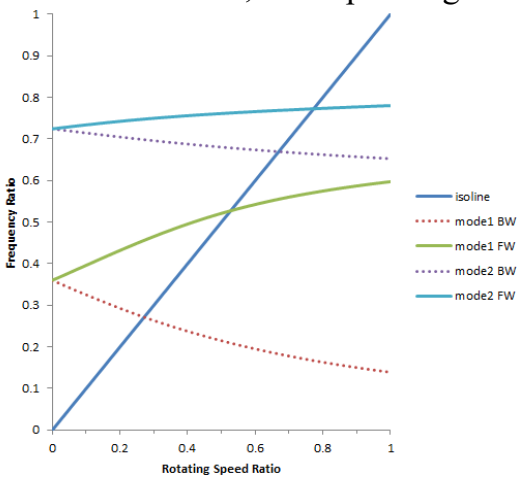

Figure 5. The Campbell diagram based on rotor model with static stiffness.
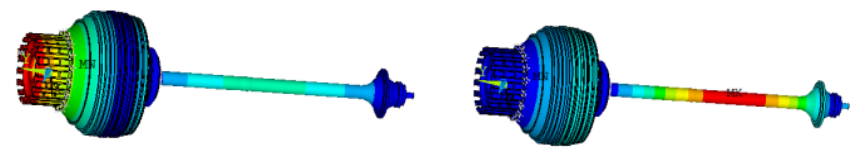

(b) Mode 2 (a) Mode 1

Figure 6. Modes of rotor. 


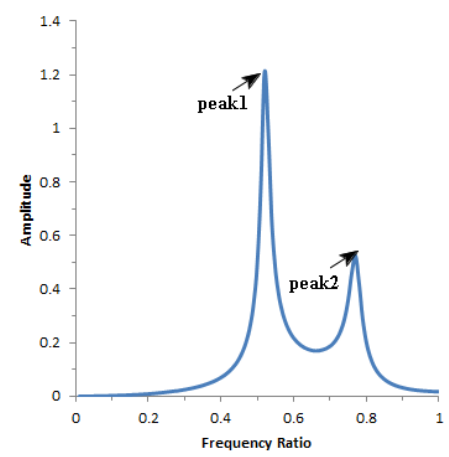

Figure 7. The displacement response amplitude based on rotor model with static stiffness.

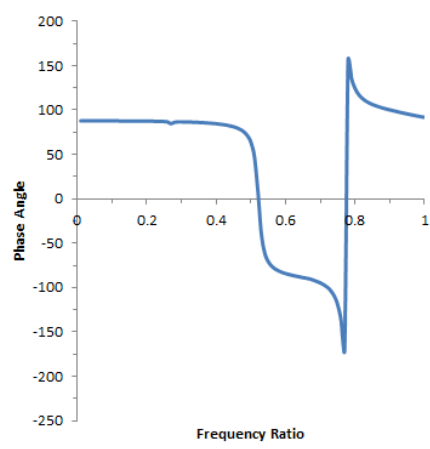

Figure 8. The displacement response phase angle based on rotor model with static stiffness.

\subsection{Rotorynamic Characteristics based on rotor model only with dynamic stiffness}

In the $K_{d}$ method, both casing stiffness and modes are considered, but coupling vibration between rotor and casing is not included. Therefore, the rotor support stiffness changes with the rotor speed, especially at the resonant mode of the casing. As a result, the frequencies in Campbell diagram change severely with the rotor speed. The Campbell diagram based on the dynamic stiffness is shown in figure 9, which shows natural frequencies of many modes change severely around 0.4 , the resonant frequency of casing. It can be seen from the Campbell diagram, the first critical speed (CS1) is 0.536 and the second critical speed (CS2) is 0.770 . The unbalance load is applied at the fan disk, and a harmonic force response analysis is performed in the frequency range of interest. The displacement response amplitude and phase angle are presented in the following figures 10 and 11 respectively. There are three distinct peaks in the exciting frequency range, the second distinct peak(peak2) and a sharp phase shift about 180 degree occurred around 0.53 , which is correspond to CS1, the third distinct peak(peak3) and a sharp phase shift about 180 degree occurred around 0.77 , which is correspond to CS2. However the first peak distinct peak and a smaller phase angle change occurred around 0.41 , which is not critical speed, and induced by dynamic stiffness only.

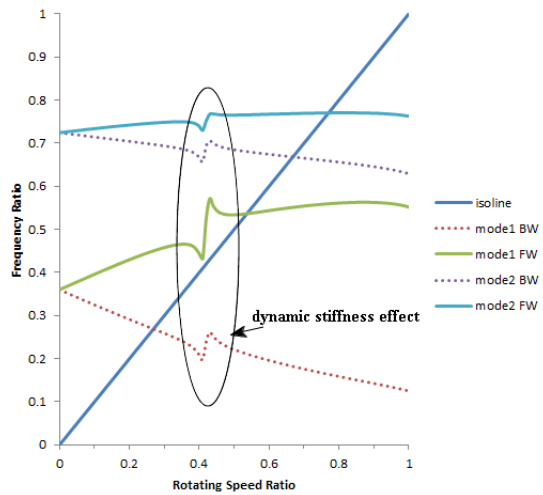

Figure 9. The Campbell diagram based on rotor model with dynamic stiffness.

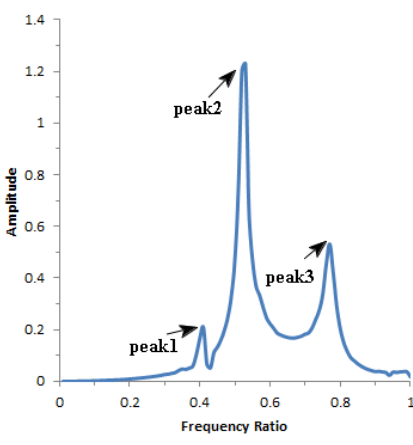

Figure 10. The displacement response amplitude based on rotor model with dynamic stiffness.

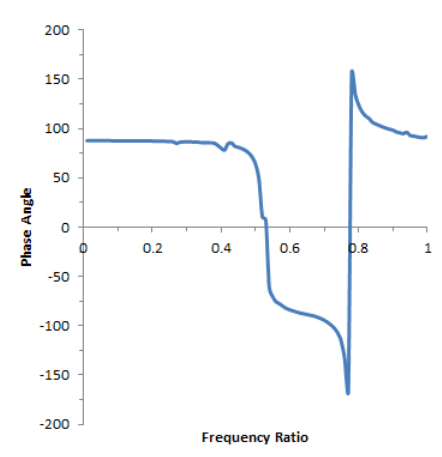

Figure 11. The displacement response phase angle based on rotor model with dynamic stiffness.

\subsection{Rotorynamic Characteristics using 3D whole engine model}

The Campbell diagram using 3D whole engine model is shown in figure.12. The whole engine model has a lot more modes than the standalone rotor model. The modes of the whole engine model include rotor modes, coupling modes between casing and rotor, as well as casing modes (mode 1-mode 5 and 
mode 10-mode 14), which do not change with the rotating speed. For clarity, casing modes are excluded and only rotor modes are retained, the modified Campbell diagram is plotted and shown in figure 13. As Figure 13 shows, there are two different mode frequencies, representing two different vibration mode shapes shown in figure 3(a) and (b). Both mode shapes are coupled vibration between the casing and rotor, meaning when the rotor is vibrating as mode 1 shown in figure 6(a), the casing may be vibrating as either of the two mode shapes. As a result, the first critical speed has two values, one is $(\mathrm{CS} 1(1)) 0.405$, the other $(\mathrm{CS} 1(2))$ is 0.540 , the second critical speed (CS2) is 0.764 . The unbalance load is applied at the fan disk, and a harmonic force response analysis is performed in the frequency range of interest. The displacement response amplitude and phase angle are presented in the following figures 14 and 15 respectively. There are three distinct peaks in the exciting frequency range, the first distinct peak (peak1) and a sharp phase shift about 180 degree occurred around 0.42 , which is correspond to CS1(1), the second distinct peak (peak2) and a sharp phase shift about 180 degree occurred around 0.54, which is correspond to $\operatorname{CS1}(2)$, the third distinct peak (peak3) and a sharp phase shift about 180 degree occurred around 0.77 , which is correspond to CS3. One can also find that one critical speed based on the whole engine model is near with the casing resonant frequency, so the engine casing needs to be improved.

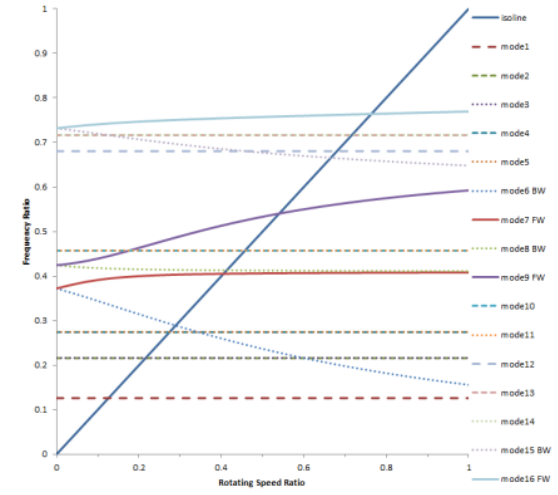

Figure 12. The preliminary Campbell diagram based on whole engine model.

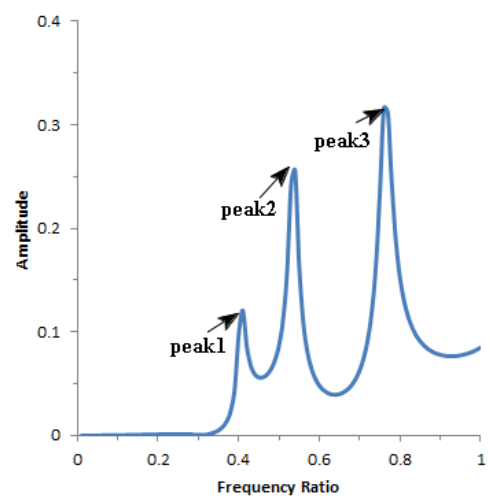

Figure 14. The displacement response amplitude based on whole engine model.

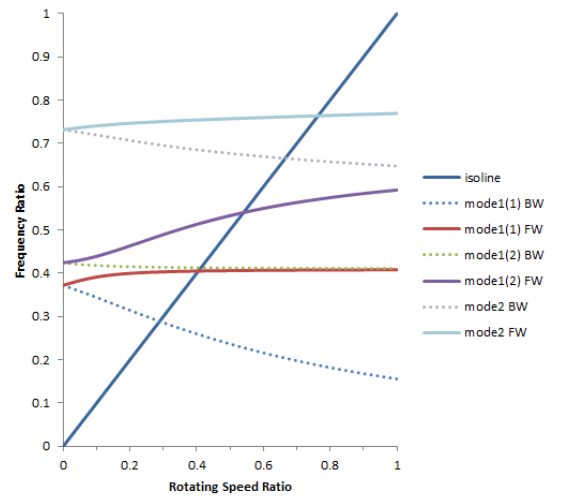

Figure 13. The modified Campbell diagram based on whole engine model.

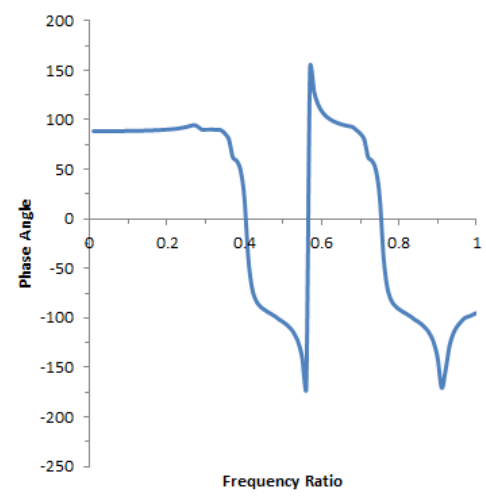

Figure 15. The displacement response phase angle based on whole engine model.

\section{Results analysis}

Table 1 shows the natural frequencies of different models at $0 \mathrm{rpm}$. It can be seen that the natural frequencies of the three models are consistent, except that the whole engine model has an additional mode frequency. The critical speeds predicted by the three models are shown in table 2 . While the critical speeds predicted by the three models are comparable, the critical speeds based on the rotor 
model with dynamic stiffness are closer to the whole engine model than the rotor model with static stiffness, since the dynamic stiffness is dynamically more representative of the whole engine model. Because of the coupling effects between the casing and rotor, there is an additional critical speed based on whole engine model. Figure 16 shows the displacement response amplitudes based on three different models. We can see that the results based on the standalone rotor models are similar, while the rotor model with dynamic stiffness has an additional peak induced by the stator casing model. The results based on whole engine model and the standalone rotor models are different in the peak values and distributions. In addition, the peak values of the whole engine model are smaller than the standalone rotor models.

Table 1. Summary of natural frequencies at $0 \mathrm{rpm}$.

\begin{tabular}{|c|c|c|c|}
\hline Mode & Rotor $\operatorname{model}\left(\boldsymbol{K}_{\boldsymbol{s}}\right)$ & $\operatorname{Rotor} \operatorname{model}\left(\boldsymbol{K}_{\boldsymbol{d}}\right)$ & Whole engine model \\
\hline 1 & 0.360 & 0.360 & 0.372 \\
\hline 2 & 0.724 & 0.724 & 0.424 \\
\hline
\end{tabular}

Table 2. Summary of critical speeds.

\begin{tabular}{|c|c|c|c|}
\hline & Rotor $\operatorname{model}\left(\boldsymbol{K}_{\boldsymbol{s}}\right)$ & $\operatorname{Rotor} \operatorname{model}\left(\boldsymbol{K}_{\boldsymbol{d}}\right)$ & Whole engine model \\
\hline $\mathrm{CS} 1$ & 0.523 & 0.536 & 0.405 \\
\hline $\mathrm{CS} 2$ & 0.760 & 0.770 & 0.540 \\
\hline
\end{tabular}

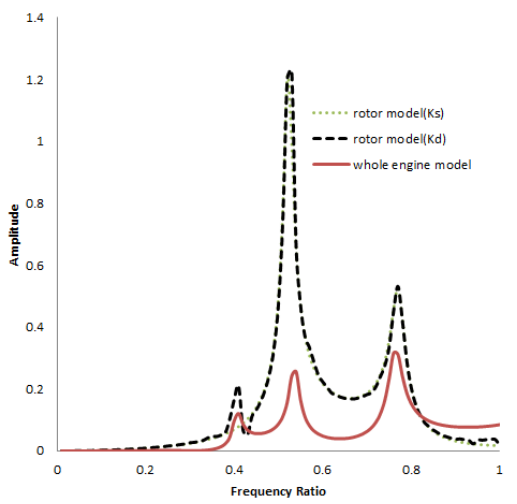

Figure 16. Summary of displacement response amplitudes.

\section{Summary and conclusions}

In this paper, 3D finite element model of an aero-engine low pressure rotor rig test system is established. The Campbell diagrams and unbalance harmonic responses are calculated respectively with static stiffness, dynamic stiffness and whole engine finite element model. The various calculation results were compared and analysed. From the simulation results, the following conclusions can be gained:

(1) The natural frequencies and critical speeds of the three models are consistent, except that the whole engine model can reveal additional mode frequencies and critical speeds induced by coupling effect between the rotor and stator casing. 
(2) The unbalance response amplitude and phase angle of the whole engine model are different from that of the standalone rotor model, in that the peak values and distributions are different, and the peak values of the whole engine model are smaller than the standalone rotor model.

(3) The critical speed calculations based on the rotor model with dynamic stiffness are closer to the whole engine model than the rotor model with static stiffness only, because the dynamic stiffness represents the whole engine model better than the rotor model only considering static stiffness.

(4) With the whole engine model, the casing modes and the coupling between the rotor and casing should be considered, so to improve the accuracy of the rotordynamic characteristics.

(5) Generally speaking, critical speed should avoid the stator casing resonant frequency. Because whole engine model can reveal more critical speeds than the standalone rotor model, whole engine model should be used in the detail design stage, and it is necessary to verify that critical speed avoids the stator casing resonant frequency.

\section{Acknowledgments}

The authors would like to thank Dr. Hu Shoufeng, Dr. Ming Cao for their guidance throughout the entire research process. The research is funded by the "Independent innovation special fund of AECC(ZZCX-2017-010)".

\section{References}

1. Chen Meng, Ma Yanhong, Liu Shuguo, Journal of Beijing University of Aeronautics and Astronautics, 33(9), 1013(2007)

2. Jeffrey J, Vannini G, Camatti M, Proceedings of ASME Turbo Expo 2006, 90481 (2006)

3. Seong Min Jeon, Hyun Duck Kwak, Suk Hwan Yoon, Journal of Propulsion and Power, 24(3), 433 (2008)

4. Hong Jie, Chen Meng and Liu Shuguo, Proceedings of ASME Turbo Expo 2007, 27162(2007)

5. Jeffrey J, Vannini G, Camatti M, Journal of Engineering for Gas Turbines and Power, 132, 082401(2010)

6. Eric Qiuli Sun, MSC 2006 Conference(2006)

7. ANSYS Documentation 2013 ANSYS Inc

8. Masaharu Shinozaki, Osamu Funatogawa and Masao Kobayashi, Proceedings of ASME Turbo Expo 2001, 0254(2001)

9. Gu Jialiu, Rotor dynamics (National Denfence Industry Press, Beijing, 1985) 